\title{
SCALAR WAVE SCATTERING OF A PROLATE SPHEROID AS A PARAMETER EXPANSION OF THAT OF A SPHERE
}

BY

THOMAS M. ACHO

University of Nigeria, Nsukka, Nigeria

Abstract. In this paper the scattering problem for the prolate spheroid,

$$
\Delta U_{p}+k^{2} U_{p}=0,
$$

is solved by way of an asymptotic parameter expansion, where the spheroid is considered a perturbation of a sphere (which has an exact solution). The error of the asymptotic approximation is then estimated.

Introduction. Generally, the problem of scattering is extremely difficult and few problems can be solved exactly. For most problems, one has to be content with some form of approximate solution. One such approximation type is that which is valid for some parameters. The sphere is one of the very few bodies for which an exact solution of the scattering problem is available, and, combined with the extreme simplicity of the shape, has necessitated the study of other convex bodies (one of which is the prolate spheroid) as a deviation from or a perturbation of the sphere.

There have been a number of studies on spheroids (oblate and prolate) by Van Buren and King [18], 1972, Senior and Uslenghi [6], 1969; Sidman and Handelman [13], 1972; and many others. However, the solution has always been in terms of spheroidal wave functions (radial and angular functions). Senior and Uslenghi (cf. [6]) expressed the scattering potential for the prolate spheroid in terms of spheroidal wave functions and later (under shape approximation) represented the scatter potential in terms of spherical functions. However, their expansion is not a parameter expansion.

In Sec. 1 we transform the spheroidal coordinates to spherical polar coordinates, in the limit, as the prolate spheroid tends to a sphere. Then we obtain the reduced wave equation for the prolate spheroid in the form

$$
\left(\Delta_{p}+k^{2}\right) U_{p}=\left(\Delta_{s}+k^{2}+\varepsilon^{2} L_{\varepsilon}\right) U_{p},
$$

where $L_{\varepsilon}$ is an operator, $\varepsilon$ is a parameter, $U_{p}$ is the scattering potential for the prolate spheroid, and $\Delta_{p}$ and $\Delta_{s}$ are the Laplacian operators for the spheroid and sphere, respectively.

Received July 13, 1990.

(C)1992 Brown University 
We look for a solution of the Helmholtz equation

$$
\left(\Delta_{p}+k^{2}\right) U_{p}=0
$$

as

$$
U_{p}=\sum_{q=0}^{\infty} \varepsilon^{2 q} Q_{2 q}(r, \theta, \psi),
$$

where $Q_{2 q}$ is to be computed.

The motivation for (1.03) stems from the asymptotic expansion for the solution $U$ of $\left(\Delta+k^{2}\right) U=0$. Sommerfeld and Rung [16] proposed the function $e^{i k \tau} \Phi$ as an asymptotic expression for $U$. Later Birkhoff in 1933 [5] generalized this procedure by proposing $U$ to be of the form

$$
e^{i k \tau} \sum_{\nu=0}^{\infty} \Phi_{\nu}(i k)^{-\nu}
$$

These asymptotic expansions, popularly known as the ray method with respect to geometric considerations, and further generalizations have been used quite often in recent years to obtain formal solutions to many problems including scattering (cf. Keller et al. [10]). Some asymptotic solutions of this form are presented in papers by Anyanwu and Keller [2, 4]; Anyanwu [3]; and Lynn and Keller [11].

In the process of developing the theory we look at the separation of variable technique of the reduced wave equation $\nabla^{2} U_{p}+k^{2} U_{p}=0$ as studied by Senior and Uslenghi in [6]. However, we consider a parameter expansion to improve their approximation (see the Appendix). Later, however, we employ the method of regular perturbation so that certain a priori conditions are avoided. The natural choice of $\varepsilon$ for our expansion is $\varepsilon=l / a$, which gives rise to (1.03). Here $a$ is the radius of the sphere to which the spheroid approximates and $l$ is one half the interfocal distance.

In Sec. 3, an error estimate of the asymptotic approximation is presented.

An application of this method [17] shows how the radiation from a spherical source near a scattering sphere could be retrieved from a situation where the spherical source is near a scattering prolate spheroid.

1. Transformation from spheroidal to spherical polar coordinates in the limit and derivation of the wave equation for the prolate spheroidal obstacle. The prolate spheroidal coordinates can be related to the Cartesian coordinates thus:

$$
\begin{aligned}
& x=l \sinh \mu \sin \nu \cos \phi, \\
& y=l \sinh \mu \sin \nu \sin \phi, \\
& z=l \cosh \mu \cos \nu,
\end{aligned}
$$

where $0 \leq \mu<\infty, 0 \leq \nu<\pi$, and $0 \leq \phi<2 \pi$.

We obtain a prolate spheroid by rotating an ellipse about the major axis (Fig. 1.1, about the $z$-axis); and an oblate spheroid by rotating about the minor axis (Fig. 1.1, about the $y$-axis).

For small values of $\mu$ prolate spheroids are rod-shaped and so can be regarded as an approximation to a wire antenna. In the case of oblate spheroids the surface $\mu=0$ is a disc $y=0, x^{2}+z^{2}=l^{2}$. 


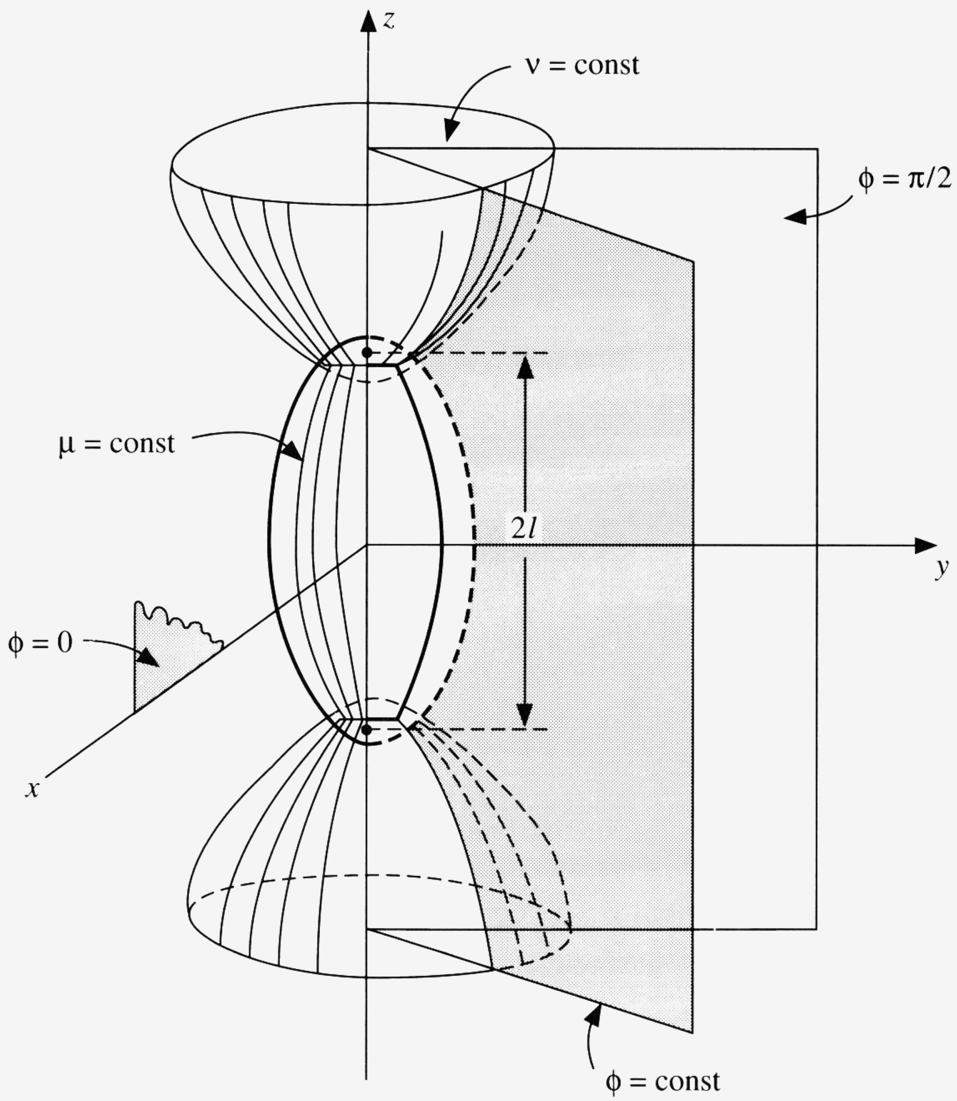

FIG. 1.1. The geometric representation of the prolate spheroid.

From the coordinate system (1.1), we obtain the Laplacian as

$$
\begin{aligned}
\nabla_{p}^{2} U_{p}=\frac{1}{h_{1} h_{2} h_{3}}\left\{\frac{\partial}{\partial \mu}\left(\frac{h_{1} h_{2}}{h_{3}} \frac{\partial U_{p}}{\partial \mu}\right)\right. & +\frac{\partial}{\partial \nu}\left(\frac{h_{3} h_{1}}{h_{2}} \frac{\partial U_{p}}{\partial \nu}\right) \\
& \left.+\frac{\partial}{\partial \phi}\left(\frac{h_{2} h_{3}}{h_{1}} \frac{\partial U_{p}}{\partial \phi}\right)\right\},
\end{aligned}
$$

where $h_{1}, h_{2}, h_{3}$ are metric coefficients given by

$$
h_{i}^{2}=\left(\frac{\partial x}{\partial u_{i}}\right)^{2}+\left(\frac{\partial y}{\partial u_{i}}\right)^{2}+\left(\frac{\partial z}{\partial u_{i}}\right)^{2}, \quad i=1,2,3,
$$

so that

$$
\begin{aligned}
\nabla_{p}^{2} U_{p}= & \frac{1}{l^{2}\left\{(\cosh \mu)^{2}-(\cos \nu)^{2}\right\}} \\
& \cdot\left\{\frac{\partial^{2} U_{p}}{\partial u^{p}}+\frac{\partial^{2} U_{p}}{\partial \nu^{2}}+\operatorname{coth} \mu \frac{\partial U_{p}}{\partial \mu}+\cos \nu \frac{\partial U_{p}}{\partial \nu}\right\} \\
& +\frac{1}{l^{2}(\sinh \mu \sin \nu)^{2}} \frac{\partial^{2} U_{p}}{\partial \phi^{2}} .
\end{aligned}
$$




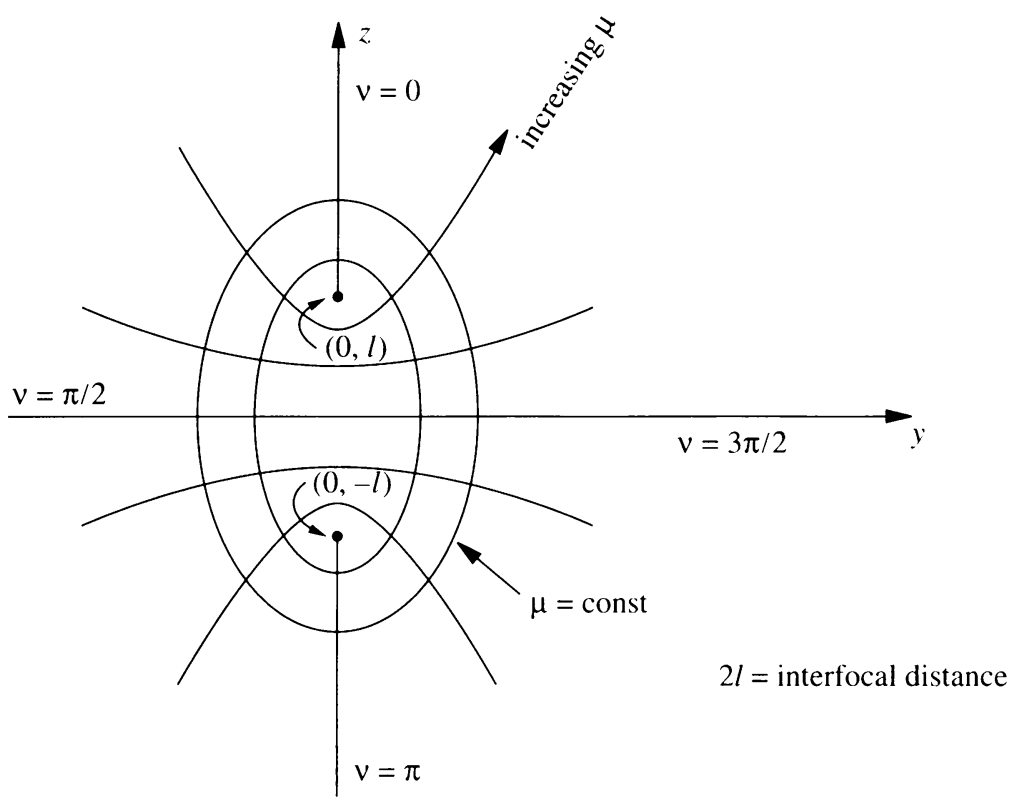

FIG. 1.2. Confocal ellipse.

Let us now look at the spherical polar coordinates given by

$$
\begin{aligned}
& x=r \sin \theta \cos \psi, \\
& y=r \sin \theta \sin \psi, \\
& z=r \cos \theta,
\end{aligned}
$$

where $0 \leq r<\infty, 0 \leq \theta \leq \pi$, and $0 \leq \psi \leq 2 \pi$.

Our immediate task would be to obtain the Laplacian of the prolate spheroid in spherical coordinates. Our expectation is that such a Laplacian would be the sum of that of a sphere and a small term. To do this, we relate the spheroidal coordinates to the polar spherical coordinates.

First, we want to find a suitable parameter that would characterize our small term. We recall that the prolate spheroid is generated by a rotation of an ellipse about its major axis. The equation of an ellipse with centre at the origin will then be

$$
\sqrt{(x-l)^{2}+y^{2}}+\sqrt{(x+l)^{2}+y^{2}}=2 a
$$

$(l=l(a, b), a$ constant $)$, that is,

$$
\frac{x^{2}}{a^{2}}+\frac{y^{2}}{a^{2}\left(1-l^{2} / a^{2}\right)}=1 .
$$

The ellipse collapses into a circle in the limit as $l / a \rightarrow 0$. We may then conclude that the prolate spheroid collapses into a sphere in the same limit $l / a \rightarrow 0$, since a sphere is generated by a circle. Thus the parameter suitable for our expansion will be $l / a$, where $l$ is one half the interfocal distance and $a$ (which is finite) is the radius of the approximating sphere. 


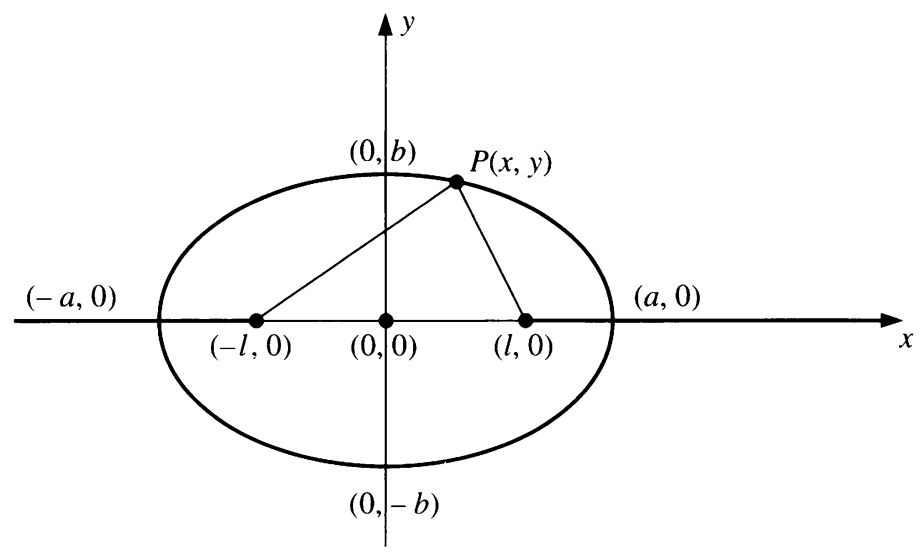

FIG. 1.3. An elliptic cross section in the Cartesian plane.

Comparing Eqs. (1.1) and (1.4), we find

$$
\frac{y}{x}=\tan \phi \quad \text { and } \quad \frac{y}{x}=\tan \psi
$$

so that

$$
\psi \rightarrow \phi
$$

We wish to relate $(\mu, \nu, \phi)$ to $(r, \theta, \psi ; \varepsilon)$ for $l / a$ small such that

$$
\begin{aligned}
& \mu=\mu(r, \theta, \psi ; \varepsilon), \\
& \nu=\nu(r, \theta, \psi ; \varepsilon), \\
& \phi=\phi(r, \theta, \psi ; \varepsilon)
\end{aligned}
$$

From (1.1) and (1.4) we have already obtained $\phi=\psi$. Also,

$$
l \sinh \mu \sin \nu=r \sin \theta, \quad l \cosh \mu \cos \nu=r \cos \theta,
$$

and for $\mu \gg 1$, looking at the dominant terms we let

$$
\begin{aligned}
e^{\mu} & =\frac{1}{\varepsilon} f_{-1}(r, \theta)+f_{0}(r, \theta)+\varepsilon^{2} f_{1}(r, \theta)+\varepsilon^{2} f_{2}(r, \theta+\cdots, \\
\nu & =g_{0}(r, \theta)+\varepsilon g_{1}(r, \theta)+\varepsilon^{2} g_{2}(r, \theta)+\varepsilon^{3} g_{3}(r, \theta)+\cdots .
\end{aligned}
$$

That is,

$$
e^{-\mu}=\frac{\varepsilon}{f_{-1}}-\frac{\varepsilon^{2} f_{0}}{f_{-1}^{2}}-\frac{\varepsilon^{3} f_{1}}{f_{-1}^{2}}-\cdots
$$


so that

$$
\begin{aligned}
\cosh \mu= & \frac{1}{2 \varepsilon} f_{-1}+\frac{1}{2} f_{0}+\frac{1}{2}\left(f_{1}+\frac{1}{f_{-1}}\right) \varepsilon \\
& +\frac{1}{2}\left(f_{2}-\frac{f_{0}}{f_{-1}^{2}}\right) \varepsilon^{2}+\frac{1}{2}\left(f_{3}-\frac{f_{1}}{f_{-1}^{2}}\right) \varepsilon^{3}+\cdots \\
\sinh \mu= & \frac{1}{2 \varepsilon} f_{-1}+\frac{1}{2} f_{0}+\frac{1}{2}\left(f_{1}-\frac{1}{f_{-1}}\right) \varepsilon+\frac{1}{2}\left(f_{2}+\frac{f_{0}}{f_{-1}^{2}}\right) \varepsilon^{2} \\
& +\frac{1}{2}\left(f_{3}+\frac{f_{1}}{f_{-1}^{2}}\right) \varepsilon^{3}+\cdots .
\end{aligned}
$$

Also,

$$
\begin{aligned}
\cos \nu= & \cos g_{0}-g_{1} \sin g \cdot \varepsilon+\left[-\frac{g_{1}}{2} \cos g_{0}-g_{2} \sin g_{0}\right] \varepsilon^{2} \\
& +\left[-g_{1} g_{2} \cos g_{0}+\sin g_{0}\left(\frac{g_{1}^{3}}{6}-g_{3}\right)\right] \varepsilon^{3}+\cdots, \\
\sin \nu= & \sin g_{0}+g_{1} \cos g_{0} \cdot \varepsilon+\left(-\frac{g_{1}^{2}}{2} \sin g_{0}+g_{2} \cos g_{0}\right) \varepsilon^{3} \\
& +\left(-g_{1} g_{2} \sin g_{0}+\left(g_{3}-\frac{g_{1}^{3}}{6}\right) \cos g_{0}\right) \varepsilon^{3}+\cdots .
\end{aligned}
$$

From (1.9) we have

$$
\begin{gathered}
a \varepsilon\left\{\frac{1}{2 \varepsilon} f_{-1}+\frac{1}{2} f_{0}+\frac{1}{2}\left(f_{1}-\frac{1}{f_{-1}}\right) \varepsilon\right. \\
\left.+\frac{1}{2}\left(f_{2}-\frac{f_{0}}{f_{-1}^{2}}\right) \varepsilon^{2}+\frac{1}{2}\left(f_{3}+\frac{f_{1}}{f_{-1}^{2}}\right) \varepsilon^{3}+\cdots\right\} \\
\cdot\left\{\sin g_{0}+g_{1} \cos g_{0} \cdot \varepsilon+\left(-\frac{g_{1}^{2}}{2} \sin g_{0}+g_{2} \cos g_{0}\right) \cdot \varepsilon^{2}\right. \\
\left.+\left(-g_{1} g_{2} \sin g_{0}+\left(g_{3}-\frac{g_{1}^{3}}{6}\right) \cos g_{0}\right) \varepsilon^{3}+\cdots\right\}
\end{gathered}
$$$$
=r \sin \theta
$$ 
and

$$
\begin{aligned}
& a \varepsilon\left\{\frac{1}{2 \varepsilon} f_{-1}\right.+\frac{1}{2} f_{0}+\frac{1}{2}\left(f_{1}+\frac{1}{f_{-1}}\right) \varepsilon \\
&+\left.\frac{1}{2}\left(f_{2}-\frac{f_{0}}{f_{-1}^{2}}\right) \varepsilon^{2}+\frac{1}{2}\left(f_{3}-\frac{f_{1}}{f_{-1}^{2}}\right) \varepsilon^{3}+\cdots\right\} \\
& \cdot\left\{\cos g_{0}-g_{1} \sin g_{0} \cdot \varepsilon+\left[-\frac{g_{1}}{2} \cos g_{0}-g_{2} \sin g_{0}\right] \varepsilon^{2}\right. \\
&\left.+\left[-g_{1} g_{2} \cos g_{0}+\sin g_{0}\left(\frac{g_{1}^{3}}{6}-g_{3}\right)\right] \varepsilon^{3}+\cdots\right\} \\
&=r \cos \theta
\end{aligned}
$$

From (1.14) and (1.15) we then obtain for,

$$
\begin{aligned}
O(1): \quad & \frac{a}{2} f_{-1} \cos g_{0}=r \cos \theta \\
& \frac{a}{2} f_{-1} \sin g_{0}=r \sin \theta \\
& g_{0}=\theta, \\
& f_{-1}=\frac{2 r}{a}, \\
& \frac{a}{2} f_{0} \cos \theta-r g_{1} \sin \theta=0 \\
& \frac{a}{2} f_{0} \sin \theta+r g_{1} \cos \theta=0 \\
\Rightarrow & f_{0}=0, \\
& g_{1}=0, \\
& \frac{a}{2} f_{1} \cos \theta-r g_{2} \sin \theta=-\frac{a^{2}}{4 r} \cos \theta, \\
O\left(\varepsilon^{2}\right):= & \frac{a}{2} f_{1} \sin \theta+r g_{2} \cos \theta=\frac{a^{2}}{4 r} \sin \theta, \\
\Rightarrow & f_{1}=-\frac{a}{2 r} \cos 2 \theta, \\
& g_{2}=\left(\frac{a}{2 r}\right)^{2} \sin 2 \theta, \\
O\left(\varepsilon^{3}\right):= & \frac{a}{2} f_{2} \cos \theta-r g_{3} \sin \theta=0, \\
& \frac{a}{2} f_{2} \sin \theta+r g_{3} \cos \theta=0, \\
& g_{3}=0, \\
& \\
& \\
&
\end{aligned}
$$

etc. 
On substitution of (1.16)-(1.19) into (1.10), we get

$$
\begin{aligned}
e^{\mu} & =\frac{2 r}{a} \cdot \frac{1}{\varepsilon}-\frac{a}{2 r} \cos 2 \theta \cdot \varepsilon+O\left(\varepsilon^{3}\right), \\
\nu & =\theta+\left(\frac{a}{2 r}\right)^{2} \sin 2 \theta \cdot \varepsilon^{2}+O\left(\varepsilon^{4}\right),
\end{aligned}
$$

and also $\phi=\psi$.

We shall now transform the reduced wave equation (1.3) using (1.20). To do this, we need the following relations which we may obtain from (1.20):

$$
\begin{aligned}
& \frac{\partial r}{\partial \mu}=r\left\{1-2\left(\frac{a}{2 r}\right)^{2} \cos 2 \theta \cdot \varepsilon^{2}+O\left(\varepsilon^{4}\right)\right\}, \\
& \frac{\partial r}{\partial \nu}=-\frac{a^{2}}{2 r} \sin 2 \theta \cdot \varepsilon^{2}+O\left(\varepsilon^{4}\right) \\
& \frac{\partial \theta}{\partial \mu}=\frac{1}{2}\left(\frac{a}{r}\right)^{2} \sin 2 \theta \cdot \varepsilon^{2}+O\left(\varepsilon^{4}\right) \\
& \frac{\partial \theta}{\partial \nu}=1-2\left(\frac{a}{2 r}\right)^{2} \cos 2 \theta \cdot \varepsilon^{2}+O\left(\varepsilon^{4}\right)
\end{aligned}
$$

Also,

$$
\begin{aligned}
& \frac{\partial^{2} r}{\partial \mu^{2}}=r\left\{1-\left(\frac{a}{r}\right)^{2} \cos 2 \theta \cdot \varepsilon^{2}+O\left(\varepsilon^{4}\right)\right\}, \\
& \frac{\partial^{2} r}{\partial \nu^{2}}=-\left(\frac{a}{r}\right)^{2} \cos 2 \theta \cdot \varepsilon^{2}+O\left(\varepsilon^{4}\right), \\
& \frac{\partial^{2} \theta}{\partial \mu^{2}}=-\left(\frac{a}{r}\right)^{2} \sin 2 \theta \cdot \varepsilon^{2}+O\left(\varepsilon^{4}\right), \\
& \frac{\partial^{2} \theta}{\partial \nu^{2}}=\left(\frac{a}{r}\right)^{2} \sin 2 \theta \cdot \varepsilon^{2}+O\left(\varepsilon^{4}\right) .
\end{aligned}
$$

We also require the relation

$$
\begin{aligned}
\frac{\partial^{2} U_{p}}{\partial u_{i}^{2}}= & \frac{\partial^{2} U_{p}}{\partial r^{2}}\left(\frac{\partial r}{\partial u_{i}}\right)^{2}+2 \frac{\partial^{2} U_{p}}{\partial r \partial \theta} \cdot \frac{\partial r}{\partial u_{i}} \frac{\partial \theta}{\partial u_{i}} \\
& +\frac{\partial^{2} U_{p}}{\partial \theta^{2}}\left(\frac{\partial \theta}{\partial u_{i}}\right)^{2}+\frac{\partial U_{p}}{\partial r} \frac{\partial^{2} r}{\partial u_{i}^{2}}+\frac{\partial U_{p}}{\partial \theta} \cdot \frac{\partial^{2} \theta}{\partial u_{i}^{2}}
\end{aligned}
$$

for $i=1,2$ and $u_{1}=\mu, u_{2}=\nu$. Also, using (1.12) and (1.13) in conjunction with (1.16)-(1.19), we have

$$
\begin{gathered}
l^{2}\left\{\cosh ^{2} \mu-\cos ^{2} \nu\right\}=r^{2}\left\{1-\left(\frac{a}{r}\right)^{2} \cos 2 \theta \cdot \varepsilon^{2}+O\left(\varepsilon^{4}\right)\right\} \\
\operatorname{coth} \mu=\frac{\cosh \mu}{\sinh \mu}=1+\frac{1}{2}\left(\frac{a}{r}\right)^{2} \cdot \varepsilon^{2}+O\left(\varepsilon^{4}\right) \\
l^{2}\left(\sinh ^{2} \mu \sin ^{2} \nu\right)=r^{2} \sin ^{2} \theta\left\{1+O\left(\varepsilon^{4}\right)\right\} \\
\cot \nu=\frac{\cos \nu}{\sin \nu}=\cot \theta\left\{1-2\left(\frac{a}{2 r}\right)^{2} \cdot \varepsilon^{2}+O\left(\varepsilon^{4}\right)\right\}
\end{gathered}
$$


and, employing (1.21) and (1.22), we obtain the following:

$$
\begin{aligned}
\frac{\partial U_{p}}{\partial \mu}= & \frac{\partial U_{p}}{\partial \theta}\left\{\frac{1}{2}\left(\frac{a}{r}\right)^{2} \sin 2 \theta \cdot \varepsilon^{2}+O\left(\varepsilon^{4}\right)\right\} \\
& +\frac{\partial U_{p}}{\partial r}\left\{r\left(1-2\left(\frac{a}{r}\right)^{2} \cos 2 \theta \cdot \varepsilon^{2}+O\left(\varepsilon^{4}\right)\right)\right\}, \\
\frac{\partial U_{p}}{\partial \nu}= & \frac{\partial U_{p}}{\partial \theta}\left\{1-2\left(\frac{a}{2 r}\right)^{2} \cos 2 \theta \cdot \varepsilon^{2}+O\left(\varepsilon^{4}\right)\right\} \\
& +\frac{\partial U_{p}}{\partial r}\left\{-\frac{a^{2}}{2 r} \sin 2 \theta \cdot \varepsilon^{2}+O\left(\varepsilon^{4}\right)\right\} .
\end{aligned}
$$

With (1.23), we get

$$
\begin{aligned}
\frac{\partial^{2} U_{p}}{\partial \mu^{2}}= & \frac{\partial^{2} U_{p}}{\partial r^{2}}\left\{r^{2}\left(1-\left(\frac{a}{r}\right)^{2} \cos 2 \theta \cdot \varepsilon^{2}+O\left(\varepsilon^{4}\right)\right)\right\} \\
& +\frac{\partial^{2} U_{p}}{\partial r \partial \theta}\left\{r\left(\frac{a}{r}\right)^{2} \sin 2 \theta \cdot \varepsilon^{2}+O\left(\varepsilon^{4}\right)\right\}+\frac{\partial^{2} U_{p}}{\partial \theta^{2}}\left\{O\left(\varepsilon^{4}\right)\right\} \\
& +\frac{\partial U_{p}}{\partial r}\left\{r\left(1-\left(\frac{a}{r}\right)^{2} \cos 2 \theta \cdot \varepsilon^{2}+O\left(\varepsilon^{4}\right)\right)\right\} \\
& +\frac{\partial U_{p}}{\partial \theta}\left\{-\left(\frac{a}{r}\right)^{2} \sin 2 \theta \cdot \varepsilon^{2}+O\left(\varepsilon^{4}\right)\right\}
\end{aligned}
$$

and

$$
\begin{aligned}
\frac{\partial^{2} U_{p}}{\partial \nu^{2}}= & \frac{\partial^{2} U_{p}}{\partial r^{2}}\left\{O\left(\varepsilon^{4}\right)\right\}+\frac{\partial^{2} U_{p}}{\partial r \partial \theta}\left\{O\left(\varepsilon^{4}\right)\right\} \\
& +\frac{\partial^{2} U_{p}}{\partial \theta^{2}}\left\{1-\left(\frac{a}{r}\right)^{2} \cos 2 \theta \cdot \varepsilon^{2}+O\left(\varepsilon^{4}\right)\right\} \\
& +\frac{\partial u_{p}}{\partial \theta}\left\{\left(\frac{a}{r}\right)^{2} \sin 2 \theta \cdot \varepsilon^{2}+O\left(\varepsilon^{4}\right)\right\} \\
& +\frac{\partial U_{p}}{\partial r}\left\{-\left(\frac{a}{r}\right)^{2} \cos 2 \theta \cdot \varepsilon^{2}+O\left(\varepsilon^{4}\right)\right\} .
\end{aligned}
$$

Now imploring (1.24)-(1.26) we then obtain the Laplacian for the prolate spheroidal 
scatterer (1.3) in the form

$$
\begin{aligned}
\nabla_{p}^{2} U_{p}= & \left\{1-\left(\frac{a}{r}\right)^{2} \cos 2 \theta \cdot \varepsilon^{2}+O\left(\varepsilon^{4}\right)\right\}^{-1} \\
& \cdot\left\{\frac{\partial^{2} U_{p}}{\partial r^{2}}+\frac{2}{r} \frac{\partial U_{p}}{\partial r}+\frac{1}{r^{2}} \frac{\partial^{2} U_{p}}{\partial \theta^{2}}+\frac{\cot \theta}{r^{2}} \frac{\partial U_{p}}{\partial \theta}\right. \\
& +\left(\frac{a}{r}\right)^{2}\left(-\cos 2 \theta \frac{\partial^{2} U_{p}}{\partial r^{2}}+\frac{\sin 2 \theta}{r} \frac{\partial^{2} U_{p}}{\partial r \partial \theta}-\frac{\cos 2 \theta}{r^{2}} \frac{\partial^{2} U_{p}}{\partial \theta^{2}}\right. \\
& \left.\left.-\frac{3(r+2)}{2 r^{2}} \cos 2 \theta \frac{\partial U_{p}}{\partial r}+\frac{1}{2 r^{2}}(1+2 \sin 2 \theta-2 \cot \theta) \frac{\partial U_{p}}{\partial \theta}\right) \varepsilon^{2}+O\left(\varepsilon^{4}\right)\right\} \\
+ & \frac{1}{r^{2} \sin ^{2} \theta} \frac{\partial^{2} U_{p}}{\partial \psi^{2}}+O\left(\varepsilon^{4}\right) .
\end{aligned}
$$

Thus the reduced wave equation for the prolate spheroidal scatterer, namely,

$$
\left(\nabla_{p}^{2}+k^{2}\right) U_{p}=0
$$

becomes

$$
\left(\nabla_{s}^{2}+k^{2}+\varepsilon^{2} L_{\varepsilon}\right) U_{p}=0
$$

where

$$
\nabla_{s}^{2}=\frac{\partial^{2}}{\partial r^{2}}+\frac{2}{r} \frac{\partial}{\partial r}+\frac{1}{r^{2}} \frac{\partial^{2}}{\partial \theta^{2}}+\frac{\cot \theta}{r^{2}} \frac{\partial}{\partial \theta}+\frac{1}{r^{2} \sin ^{2} \theta} \frac{\partial^{2}}{\partial \psi^{2}}
$$

and

$$
\begin{aligned}
L_{\varepsilon}=\left(\frac{a}{r}\right)^{2} & \left\{-\cos 2 \theta \frac{\partial^{2}}{\partial r^{2}}+\frac{1}{r} \sin 2 \theta \frac{\partial^{2}}{\partial r \partial \theta}-\frac{\cos 2 \theta}{r^{2}} \frac{\partial^{2}}{\partial \theta^{2}}\right. \\
& -\frac{3(r+2)}{2 r^{2}} \cos 2 \theta \frac{\partial}{\partial r}+\frac{1}{2 r^{2}}(1+2 \sin 2 \theta-2 \cot \theta) \frac{\partial}{\partial \theta} \\
& \left.+\frac{1}{r^{2}}\left(\csc ^{2} \theta-2\right) \frac{\partial^{2}}{\partial \psi^{2}}+k^{2} \cos 2 \theta\right\}+O\left(\varepsilon^{2}\right) .
\end{aligned}
$$

It is obvious from (1.29) that we retrieve the reduced wave equation for the spherical scatterer, as expected, when we set $\varepsilon=0$.

It is worth mentioning that the independent variable $U_{p}$ under the transformation (1.20) is invariant, consistent with the geometrical theory of wave propagation. This can easily be verified by substituting $U_{p}=J^{\alpha} U_{p}^{*}$ into (1.28), where $J$, the Jacobian of the transformation, is given by

$$
J=\frac{1}{\rho}\left\{1+2\left(\frac{a}{2 \rho}\right)^{2} \cos 2 \theta \cdot \varepsilon^{2}+O\left(\varepsilon^{4}\right)\right\},
$$

and $U_{p}^{*}$ is the transformed independent variable. As $\varepsilon \rightarrow 0$, we should obtain the Helmholtz equation for the spherical scatterer and such is the case when $\alpha \equiv 0$. 
2. The asymptotic expansion of the scattered field for the prolate spheroidal scatterer. The scattered field $U_{p}$ of the prolate spheroidal scatterer may be expressed as

$$
U_{p} \sim \sum_{q=0}^{\infty} \varepsilon^{2 q} Q_{2 q}(r, \theta, \psi)
$$

The motivation for this choice of $U_{p}$ was stated earlier in the introduction. The scattered field $U_{s}$ by the sphere for an incident plane wave in the direction $\theta_{0}$ and $\psi_{0}$ has the exact value given by [6, p. 358]

$$
\begin{array}{r}
U_{s}=-\sum_{n=0}^{\infty} \sum_{m=0}^{\infty} i^{n} \varepsilon_{m} \frac{(n-m) !}{(n+m) !}(2 n+1) \frac{j_{n}(k a)}{h_{n}^{(1)}(k a)} h_{n}^{(1)}(k r) \\
\cdot P_{n}^{m}(\cos \theta) P_{n}^{m}\left(\cos \theta_{0}\right) \cos m\left(\psi-\psi_{0}\right),
\end{array}
$$

and the expansion for the incident wave is

$$
\begin{array}{r}
U_{i}=-\sum_{m=0}^{\infty} \sum_{n=m}^{\infty} i^{n} \varepsilon_{m} \frac{(n-m) !}{(n+m) !} j_{n}(k r) P_{n}^{m}(\cos \theta) \\
\cdot P_{n}^{m}\left(\cos \theta_{0}\right) \cos m\left(\psi-\psi_{0}\right),
\end{array}
$$

where $\varepsilon_{m}=$ Neumann symbol $\left(\varepsilon_{0}=1, \varepsilon_{m}=2\right.$ for $\left.m=1,2, \ldots\right)$ and the other symbols have their usual meanings. Equation (1.29) may be written as

$$
\left(\Delta_{s}+k^{2}+\varepsilon^{2} L_{\varepsilon}\right)\left[Q_{0}+\varepsilon^{2} Q_{2}+\varepsilon^{4} Q_{4}+\cdots\right]=0,
$$

that is,

$$
\left(\Delta+k^{2}+\varepsilon^{2} L_{\varepsilon}\right) Q_{0}+\varepsilon^{2}\left(\Delta+k^{2}+\varepsilon^{2} L_{\varepsilon}\right) Q_{2}+\varepsilon^{4}\left(\Delta+k^{2}+\varepsilon^{2} L_{\varepsilon}\right) Q_{4}+\cdots=0,
$$

where the subscript $s$ on the $\Delta$ (that is, $\nabla^{2}$ ) operator has been dropped for the sake of brevity. We shall now employ the method of regular perturbation on Eq. (2.5). The constant terms (i.e., terms of order unity) are

$$
\left(\Delta_{s}+k^{2}\right) Q_{0}=0 \text {. }
$$

The above Eq. (2.6) is the Helmholtz equation for the spherical scatterer. Considering the fact that $U_{p} \rightarrow U_{s}$ when $\varepsilon \rightarrow 0$ in Eq. (2.1), it becomes obvious that the most suitable choice for $Q_{0}$ is

$$
Q_{0}=U_{s} \text {. }
$$

We now consider terms of order $\varepsilon^{2}$ in $(2.5)$ :

$$
\left(\Delta+k^{2}\right) Q_{2}=-L_{\varepsilon^{0}} Q_{0},
$$

where $L_{\varepsilon^{2 n}}(n=0,1,2, \ldots)$ represent terms in the operator $(1.30 \mathrm{~b})$ of up to order $2 n$ (that is, terms of $O\left(\varepsilon^{2 n}\right)$ ), so that generally we obtain $Q_{2 n}$ from

$$
\left(\Delta+k^{2}\right) Q_{2 n}=-\sum_{i=0}^{n-1} L_{\varepsilon^{2 i}} Q_{2(n-1-i)} .
$$


Let us apply the boundary condition on the surface, namely

$$
U_{i}+U_{p}=0
$$

that is,

$$
U_{i}=\sum_{q=0}^{\infty} \varepsilon^{2 q} Q_{2 q}(r, \theta, \psi)=0 .
$$

Now, since on the surface $r=a$ and $Q_{0}=U_{s}$ (where $U_{i}$ and $U_{s}$ are defined in (2.2) and (2.3)), it follows that

$$
\left[Q_{2 n}\right]_{\text {surface }}=0 .
$$

Also $U_{p}$ satisfies the radiation condition, namely

$$
U_{p} \sim \frac{c}{r} \quad \text { as } r \rightarrow \infty,
$$

where $c$ is a constant. We shall now construct a suitable Green's function for our problem, taking into consideration the boundary condition at the surface and the radiation condition. The Green's function would satisfy the homogeneous equation

$$
\Delta G+k^{2} G=0 \quad \text { except at }\left(r_{0}, \theta_{0}, \psi_{0}\right),
$$

and for $R=\left|r-r_{0}\right|$

$$
G \sim \frac{1}{4 \pi R} \quad \text { as } R \rightarrow 0 \text { (i.e., } r \rightarrow r_{0} \text { ) }
$$

and

$$
G \sim \frac{c}{r} \quad \text { as } r \rightarrow \infty(c=\text { constant }) .
$$

With a Green's function determined satisfying the above conditions (2.14)-(2.16), we may then write the solution of (2.9) as

$$
\begin{aligned}
Q_{2 q}\left(r_{0}, \theta_{0}, \psi_{0}\right)=\int_{0}^{2 \pi} \int_{0}^{\pi} \int_{r_{0}}^{\infty} G\left(r, \theta, \psi ; r_{0}, \theta_{0}, \psi_{0}\right) \\
\cdot\left(\sum_{i=0}^{q-1} L_{\varepsilon^{2 l}} Q_{2(q-1-i)}\right) r^{2} \sin \theta d r d \theta d \psi .
\end{aligned}
$$

The Green's function in spherical coordinates for the reduced wave equation (Morse and Feshbach [12, p. 887]) is

$$
\begin{aligned}
& G=\frac{e^{i k R}}{4 \pi R}=\frac{i k}{4 \pi} \sum_{\substack{n=0 \\
m=0}}^{\infty} \varepsilon_{m}(2 n+1) \frac{(n-m) !}{(n+m) !} \cos m\left(\psi-\psi_{0}\right) \\
& \cdot P_{n}^{m}(\cos \theta) P_{n}^{m}(\cos \theta) j_{n}\left(k r_{0}\right) h_{n}(k r), \quad r \geq r_{0},
\end{aligned}
$$

where $G=G\left(r, \theta, \psi ; r_{0}, \theta_{0}, \psi_{0}\right)$. 
Let us now obtain $Q_{2}$. To do this we shall first of all compute $L_{\varepsilon^{0}} Q_{0}$ :

$$
\begin{aligned}
L_{\varepsilon^{0}} Q_{0}=\left(\frac{a}{r}\right)^{2}\left\{k^{2}\right. & \cos 2 \theta-\frac{m^{2}}{r^{2}}\left(\csc ^{2} \theta-2\right) \\
& -\cos 2 \theta\left(h_{n}^{(1)}(k r)\right)^{-1} \frac{d^{2}}{d r^{2}}\left[h_{n}^{(1)}(k r)\right] \\
& +\frac{\sin 2 \theta}{r}\left(P_{n}^{m}(\cos \theta) h_{n}^{(1)}(k r)\right)^{-1} \frac{d}{d r}\left[h_{n}^{(1)}(k r)\right] \frac{d}{d \theta}\left[P_{n}^{m}(\cos \theta)\right] \\
& -\frac{\cos 2 \theta}{r^{2}}\left(P_{n}^{m}(\cos \theta)\right)^{-1} \frac{d^{2}}{d \theta^{2}}\left[P_{n}^{m}(\cos \theta)\right] \\
& -\frac{3(r+2)}{2 r^{2}} \cos 2 \theta\left(h_{n}^{(1)}(k r)\right)^{-1} \frac{d}{d r}\left[h_{n}^{(1)}(k r)\right] \\
& +\frac{1}{2 r^{2}}(1+2 \sin 2 \theta-2 \cot \theta)\left(P_{n}^{m}(\cos \theta)\right)^{-1} \\
& \left.\cdot \frac{d}{d \theta}\left[P_{n}^{m}(\cos \theta)\right]\right\} U_{s}
\end{aligned}
$$

where

$$
\begin{aligned}
& \frac{d}{d \theta}\left[P_{n}^{m}(\cos \theta)\right]= n \cot \theta P_{n}^{m}(\cos \theta)-\frac{(n+m)}{\sin \theta} P_{n-1}^{m}(\cos \theta) \\
& \frac{d^{2}}{d \theta^{2}}\left[P_{n}^{m}(\cos \theta)\right]=\left(n \cot ^{2} \theta+n+n^{2} \cot ^{2} \theta \sin ^{2} \theta\right) P_{n}^{m}(\cos \theta) \\
&+\left((n-1) \sin ^{2} \theta \cot \theta-(n+m) \cos \theta\right. \\
&-(n+m) \cot \theta \csc \theta) P_{n-1}^{m}(\cos \theta) \\
&-(n+m-1) \cos \theta P_{n-2}^{m}(\cos \theta) \\
& \frac{d}{d r}\left[h_{n}^{(1)}(k r)\right]=k h_{n-1}^{(1)}(k r)-\frac{(n+1)}{r} h_{n}^{(1)}(k r)
\end{aligned}
$$

and

$$
\begin{aligned}
\frac{d^{2}}{d r^{2}}\left[h_{n}^{(1)}(k r)\right]= & k^{2} h_{n-2}^{(1)}(k r)-\frac{k}{2}(n+(n+1) k) h_{n-1}^{(1)}(k r) \\
& +\frac{k(n+1)(n+2)}{r^{2}} h_{n}^{(1)}(k r) .
\end{aligned}
$$

Using (2.18) and (2.19) we may now evaluate (2.17) for $Q_{2}$. We shall employ the trigonometric expansions for the associated Legendre polynomials which we may write as (see p. 335 of [1])

$$
P_{n}^{m}(\cos \theta)=S_{k}(m, n)(\sin \theta)^{m} \sin [(m+n+2 k+1) \theta], \quad k=0,1, \ldots,
$$

where

$$
S_{k}(m, n)=\pi^{-1 / 2} 2^{m+1} \frac{\Gamma(m+n+1)}{\Gamma(n+3 / 2)} \sum_{k=0}^{\infty} \frac{(m+1 / 2)_{k}(m+n+1)_{k}}{k !(n+3 / 2)_{k}} .
$$


Now, imploring [1] and [7], we may then integrate to obtain

$$
\begin{aligned}
& Q_{2}=\frac{k \pi}{4 a} \sum_{\substack{n=0 \\
m=0}}^{\infty} \frac{i^{n+1}}{2^{2 m}} \varepsilon_{m}\left\{(2 n+1) \frac{(n-m) !}{(n+m) !} P_{n}^{m}\left(\cos \theta_{0}\right) \cdot S_{p}(m, n) j_{n}(k a)\right\}^{2} h_{n}^{(1)}(k a) \\
& \cdot\{-16\left(\frac{m^{2}}{(2 n+3)}+\frac{n}{2 n+3}\right)\left(\begin{array}{c}
2(m-1) \\
m-1
\end{array}\right) \\
&+\left(\frac{15(k a)^{2}}{2(2 n+1)}-\frac{m^{2}}{2(2 n+3)}+\frac{15 k(n+1)(n+2)}{2(2 n+3)}+\frac{8 n(n+1)}{(2 n+3)}\right. \\
&\left.-\frac{15 n}{(2 n+3)}-\frac{4 n^{2}}{(2 n+3)}-\frac{15(n+1)}{2}\left[\frac{3}{2(2 n+3)}-\frac{1}{2 n+4}\right]+\frac{8 n}{2 n+3}\right) \\
&\left.\cdot\left(\begin{array}{c}
2 m \\
m
\end{array}\right)-\frac{2 n^{2}}{(2 n+3)}\left(\begin{array}{c}
2(m+1) \\
m+1
\end{array}\right)\right\}, \quad(2.21)
\end{aligned}
$$

where

$$
\left(\begin{array}{c}
2 m \\
m
\end{array}\right)=\frac{2 m(2 m-1)(2 m-2) \cdots(2 m-m+1)}{1 \cdot 2 \cdot 3 \cdots m},
$$

$S_{p}(m, n)$ is as given in $(2.20 \mathrm{~b})$, and all the other symbols have their usual meanings.

With $Q_{2}$ determined the scattered field $U_{p}$ of the prolate spheroidal scatterer may then be given as in (2.1).

3. Error estimate in the asymptotic approximation. We shall now estimate the error in our asymptotic approximation. For convenience we shall simply write $U_{p}$ as $U$, so that

$$
U=\sum_{q=0}^{\infty} \varepsilon^{2 q} Q_{2 q}
$$

Now let

$$
U_{N}=\sum_{q=0}^{N} \varepsilon^{2 q} Q_{2 q}
$$

and let the error be

$$
R_{N}=U_{N}-U
$$

We invoke Eq. (1.29), which is

$$
\left(\Delta+k^{2}+\varepsilon^{2} L_{\varepsilon}\right) U=0,
$$

so that

$$
\left(\Delta+k^{2}+\varepsilon^{2} L_{\varepsilon}\right)\left(U_{N}-R_{N}\right)=0
$$

or

$$
\left(\Delta+k^{2}+\varepsilon^{2} L_{\varepsilon}\right) U_{N}=\left(\Delta+k^{2}+\varepsilon^{2} L_{\varepsilon}\right) R_{N} .
$$

The left-hand side of this equation yields

$$
\left(\Delta+k^{2}+\varepsilon^{2} L_{\varepsilon}\right) U_{N}=\left(\Delta+k^{2}+\varepsilon^{2} L_{\varepsilon}\right)\left\{\sum_{q=0}^{N} \varepsilon^{2 q} Q_{2 q}\right\} .
$$


Employing (3.1) we write

$$
\left(\Delta+k^{2}+\varepsilon^{2} L_{\varepsilon}\right) U_{N}=-\left(\Delta+k^{2}\right)\left\{\sum_{q=N+1}^{\infty} \varepsilon^{2 q} Q_{2 q}\right\}-\varepsilon^{2} L_{\varepsilon}\left\{\sum_{q=N+1}^{\infty} \varepsilon^{2 q} Q_{2 q}\right\}
$$

so that, using (2.9), we get

$$
\left(\Delta+k^{2}+\varepsilon^{2} L_{\varepsilon}\right) U_{N}=\sum_{q=N+1}^{\infty} \varepsilon^{2 q} L_{\varepsilon^{2(q-1)}} Q_{2(q-1)}-\sum_{q=N+1}^{\infty} \varepsilon^{2(q+1)} L_{\varepsilon} Q_{2 q},
$$

that is,

$$
\left(\Delta+k^{2}+\varepsilon^{2} L\right) U_{N}=\varepsilon^{2(N+1)} L_{\varepsilon^{2 N}} Q_{2 N}+\sum_{q=N+1}^{\infty} \varepsilon^{2(q+1)}\left(L_{\varepsilon^{2 q}}-L_{\varepsilon}\right) Q_{2 q}
$$

or

$$
\left(\Delta+k^{2}+\varepsilon^{2} L_{\varepsilon}\right) U_{N}=\left(L_{\varepsilon^{2 N}} Q_{2 N}\right) \cdot \varepsilon^{2(N+1)}+O\left(\varepsilon^{2(N+2)}\right) .
$$

On substitution into (3.5) we obtain

$$
\left(\Delta+k^{2}\right) R_{N}=-\varepsilon^{2} L_{\varepsilon} R_{N}+\left(L_{\varepsilon^{2 N}} Q_{2 N}\right) \cdot \varepsilon^{2(N+1)}+O\left(\varepsilon^{2(N+2)}\right),
$$

but

or

$$
L_{\varepsilon} R_{N}=L_{\varepsilon}\left\{\sum_{q=N+1}^{\infty} \varepsilon^{2 q} Q_{2 q}\right\}=\sum_{q=N+1}^{\infty} \varepsilon^{2 q} L_{\varepsilon} Q_{2 q}
$$

$$
L_{\varepsilon} R_{N}=\varepsilon^{2(N+1)} L_{\varepsilon^{0}} Q_{2 N}+O\left(\varepsilon^{2(N+1)}\right)
$$

so that from (3.9) we then get

$$
\left(\Delta+k^{2}\right) R_{N}=\left(L_{\varepsilon^{2 N}} Q_{2 N}\right) \cdot \varepsilon^{2(N+1)}+O\left(\varepsilon^{2(N+2)}\right) .
$$

This is a reduced wave equation in $R_{N}$ for which $\Delta$ is the spherical operator. We may then obtain $R_{N}$ as

$$
R_{N}=\varepsilon^{2(N+1)} \int_{\Omega} G\left(r, \theta, \psi ; r^{\prime}, \theta^{\prime}, \psi^{\prime}\right)\left[L_{\varepsilon^{2 N}} Q_{2 N}\right] d \Omega+O\left(\varepsilon^{2(N+2)}\right)
$$

so that

$$
\left|R_{N}\right| \leq \varepsilon^{2(N+1)} \int_{\Omega}\left|G\left(r, \theta, \psi ; r^{\prime}, \theta^{\prime}, \psi^{\prime}\right)\right|\left|L_{\varepsilon^{2 N}} Q_{2 N}\right| d \Omega+\left|O\left(\varepsilon^{2(N+2)}\right)\right| .
$$

But

and from (2.18)

$$
\left|S_{N}\right|=\left|L_{\varepsilon^{2 N}} Q_{2 N}\right|=O(1)
$$

$$
\left|G\left(r, \theta, \psi ; r^{\prime}, \theta^{\prime}, \psi^{\prime}\right)\right|=O(1) .
$$

Thus from (3.13) we may conclude that

$$
R_{N}=O\left|\varepsilon^{2(N+1)}\right| \text {. }
$$

This error function suggests that the error would be quite small for $\varepsilon \rightarrow 0$, i.e., $O\left(\varepsilon^{4}\right)$ if the first two terms of the expansion in (2.1) are taken as an approximation. 
4. Appendix. The scattering potential for the prolate spheroid that departs only infinitesimally from the sphere as a perturbation of the solution for a sphere was given in [6]. Separation of variables of (1.28) for

$$
\frac{l^{2} \cos ^{2} \theta}{r^{2}}=\frac{l^{2}}{a^{2}} \quad\left(=\varepsilon^{2}\right),
$$

where $\varepsilon$ is a small parameter, yields a result that is close to the result in [6, p. 437]. The raison d'etre for setting $l^{2} \cos ^{2} \theta / r^{2}=\varepsilon^{2}$ is that $\left|\cos ^{2} \theta\right| \leq 1$ and on the spherical surface $(r=a)$ and outside the body (sphere)

$$
\frac{l^{2} \cos ^{2} \theta}{r^{2}}<\frac{l^{2}}{a^{2}}=\varepsilon^{2} \text {. }
$$

With this approximation, we assume that the solution of (1.28) is of the form

$$
U_{p}=R(r) T(\psi) H(\theta)
$$

so that on separating (1.28) we get

$$
\begin{aligned}
& R^{\prime \prime}+\frac{2}{r} R^{\prime}+\left[k^{2}\left(1-\varepsilon^{2}\right)-\frac{n(n+1)}{r^{2}}\right] R=0, \\
& H^{\prime \prime}+\cot \theta H^{\prime}+\left[n(n+1)-\frac{m^{2}}{\sin ^{2} \theta}\right] H=0, \\
& T^{\prime \prime}+\frac{m^{2}}{(1+\varepsilon)} T=0 .
\end{aligned}
$$

Solutions to these equations take the form

$$
\begin{aligned}
H & =P_{n}^{m}(\cos \theta), \\
T & =a_{m} \cos m \rho \psi+b_{m} \sin m \rho \psi, \\
R & =c_{n} j_{n}\left(k_{p} r\right)+d_{n} h_{n}^{(1)}\left(k_{p} r\right),
\end{aligned}
$$

respectively, where the symbols have their usual meaning and

$$
\rho=(1+\varepsilon)^{-1 / 2}, \quad k_{p}=k\left(1-\varepsilon^{2}\right)^{1 / 2} .
$$

Thus the scattered potential $U_{p}$ becomes

$$
U_{p}=\sum_{m=0}^{\infty} \sum_{n=m}^{\infty} a_{n m} h_{n}\left(k_{p} r\right) P_{n}^{m}\left(\cos \theta_{0}\right) P_{n}^{m}(\cos \theta) \cos m \rho\left(\psi-\psi_{0}\right) .
$$

But the expansion of the incident wave for the spheroid is given by

$$
\begin{gathered}
U^{\underline{i}}=\sum_{m=0}^{\infty} \sum_{n=m}^{\infty} i^{n} \varepsilon_{m}(2 n+1) \frac{(n-m) !}{(n+m) !} j_{n}\left(k_{p} r\right) P_{n}^{m}\left(\cos \theta_{0}\right) \\
\cdot P_{n}^{m}(\cos \theta) \cos m \rho\left(\psi-\psi_{0}\right) .
\end{gathered}
$$

On application of the Dirichlet boundary condition $U^{\underline{i}}+U_{p}=0$ on the surface, we obtain with the use of the orthongality condition for trigonometric and Legendre functions that

$$
a_{m n}=\varepsilon_{m} i^{n}(2 n+1) \frac{(n-m) !}{(n+m) !} \frac{j_{n}\left(k_{p} a\right)}{h_{n}\left(k_{p} a\right)} .
$$


Using (4.5) and the expansion for the spherical functions $j_{n}\left(k_{p} a\right)$ and $h_{n}\left(k_{p} a\right)$ we obtain

$$
\begin{aligned}
& a_{m n} h_{n}^{(1)}\left(k_{p} r\right)= \varepsilon_{m} i^{n} \frac{(n-m) !}{(n+m) !} \frac{j_{n}(k a)}{h_{n}^{(1)}(k a)} \\
& \cdot\left\{1+\frac{\varepsilon}{2 h_{n}^{(1)}(k a)}\left[\frac{(k a) j_{n+1}(k a) h_{n}^{(1)}(k a)}{j_{n}(k a)}-(k a) j_{n+1}(k a)\right.\right. \\
&+i\left(2 y_{n-1}(k a)-y_{n}(k a)\right)+n h_{n}^{(1)}(k a) \\
& \frac{(k r) j_{n}(k r) h_{n}^{(1)}(k a)}{h_{n}^{(1)}(k r)}+i \frac{y_{n}(k r) h_{n}^{(1)}(k a)}{h_{n}^{(1)}(k r)} \\
&\left.\left.-i \frac{y_{n-1}(k r) \cdot h_{n}^{(1)}(k a)}{h_{n}^{(1)}(k r)}\right]\right\}+O\left(\varepsilon^{2}\right),
\end{aligned}
$$

which on substitution into (4.6) gives $U_{p}$.

Conclusion. The method developed in this work has been applied to other work by the author [17]. Apart from the usual powerful advantage of a parameter expansion, this method has a special advantage over the traditional methods in that the scattering potentials for the spheroid may be obtained in terms of simpler functions (spherical functions) instead of the more complicated spheroidal functions. Furthermore, scattering and diffraction problems whose solutions are known for the sphere can very easily be transcribed to the spheroidal shapes, and vice versa through a careful limiting process. The scattered potential $U_{p}$ for the prolate spheroid could stand for any other wave function provided linearity in the initial transformation is maintained.

Acknowledgment. My profound gratitude goes to Professor J. C. Amazigo for his unrelenting assistance towards obtaining the much desired coordinate transformations in the work. I also wish to thank the referee for his comments which have greatly improved the readability of this paper.

\section{REFERENCES}

[1] M. Abramowitz and I. A. Stegun, Handbook of Mathematical Functions, Dover, New York, 1965.

[2] D. U. Anyanwu and J. B. Keller, Asymptotic solution of eigenvalue problems for second-order ordinary differential equations, Comm. Pure Appl. Math. 28, 753-763 (1975)

[3] D. U. Anyanwu, Uniform asymptotic solutions of nonhomogeneous differential equations with turning points, SIAM J. Math. Anal. 8, 710-719 (1977)

[4] D. U. Anyanwu and J. B. Keller, Asymptotic solutions of higher order differential equations with several turning points, and application to wave propagation in slowly varying wave guides, Comm. Pure Appl. Math. 31, 107-121 (1978)

[5] G. D. Birkhoff, Quantum mechanics and asymptotic series, Bull. Amer. Math. Soc. 39, 681-700 (1933)

[6] J. J. Bowman et al., Electromagnetic and Acoustic Scattering by Simple Shapes, North-Holland, Amsterdam, 1969 
[7] I. S. Gradshteyn and I. W. Ryzhik, Table of Integrals, Series and Products, Academic Press, New York, 1963

[8] A. C. Hewson, An Introduction to the Theory of Electromagnetic Waves, Longman, London 1970

[9] J. B. Keller and R. M. Lewis, Asymptotic methods for partial differential equations, New York Univ., Courant Inst. Math. Sci., Electromagnetic Res., Rep. No. tM-194, 1964

[10] J. B. Keller et al., Asymptotic solution of some diffraction problems, Comm. Pure Appl. Math. 9, 207-266 (1956)

[11] R. Y. S. Lynn and J. B. Keller, Uniform asymptotic solutions of second order linear ordinary differential equations with turning points, Comm. Pure Appl. Math. 23, 379-408 (1970)

[12] P. M. Morse and H. Feshbach, Methods of Mathematical Physics, McGraw-Hill, New York, 1953

[13] Robert D. Sidman and George H. Handelman, Motion of a spherical obstacle generated by plane or spherical acoustic waves, J. Acoust. Soc. Amer. (3) 52, 923-927 (1972)

[14] Robert D. Sidman, Scattering of acoustic waves by a prolate spheroidal obstacle, J. Acoust. Soc. of Amer. (3) 52, 879-883 (1972)

[15] Seymour Stein, Addition theorems for spherical wave functions, Quart. Appl. Math. 19, 15-24 (1961)

[16] A. Sommerfeld and J. Rung, Anvedung der Vektorrechung auf die Grundlages der geometrischen Optik, Ann. Physik 35, 277-298 (1911)

[17] Thomas M. Acho, Scattering by a fluid prolate spheroid of radiation from a nearby spherical acoustic source, submitted for publication

[18] A. L. Van Buren and B. J. King, Acoustic radiation from two spheroids, J. Acoust. Soc. Amer. (1) 52, 364-372 (1972)

[19] William Thompson, Acoustic radiation from a spherical source embedded eccentrically within a fluid sphere, J. Acoust. Soc. Amer. 54, 1694-1707 (1973)

[20] William Thompson, Radiation from a spherical acoustic source near a scattering sphere, J. Acoust. Soc. Amer. 60, 781-787 (1976) 